\title{
Patients with inflammatory bowel disease have higher abdominal adiposity and less skeletal mass than healthy controls
}

\author{
Teresa Barroso a, Fiona Conway ${ }^{a}$, Sari Emel ${ }^{b}$, Donald McMillana , David Youngc, Hedvig Karteszib, \\ Daniel R. Gaya ${ }^{d}$, Konstantinos Gerasimidis ${ }^{a}$
}

University of Glasgow, Glasgow Royal Infirmary; Glasgow Royal Infirmary; University of Strathclyde, Glasgow, UK

\section{Abstract}

${ }^{a}$ School of Medicine, College of Medical, Veterinary and Life Sciences, University of Glasgow, Glasgow Royal Infirmary (Teresa Barroso, Fiona Conway, Donald McMillan, Konstantinos Gerasimidis); ${ }^{b}$ Department of Radiology, Glasgow Royal Infirmary (Sari Emel, Hedvig Karteszi); 'Department of Mathematics and Statistics, University of Strathclyde (David Young); ${ }^{\mathrm{d}}$ Gastroenterology Unit, Glasgow Royal Infirmary

(Daniel R. Gaya), Glasgow, UK

Conflict of Interest: None

Correspondence to: Dr Konstantinos Gerasimidis, Human Nutrition, School of Medicine College of Medical, Veterinary and Life Sciences, University of Glasgow, New Lister Building, Glasgow Royal Infirmary, Glasgow, UK, G31 2ER,

e-mail: konstantinos.gerasimidis@glasgow.ac.uk

Received 14 January 2018; accepted 17 April 2018; published online 25 May 2018

DOI: https://doi.org/10.20524/aog.2018.0280

\section{Introduction}

Alterations in the body composition of patients with inflammatory bowel disease (IBD) have been described previously in studies using bioelectrical impedance analysis and dual X-ray absorptiometry [1]. Conventional body composition constants, such as hydration level of lean mass, have been developed in health; however, since these are different in IBD patients [2], methods that consider these constants in the calculation of body composition (e.g., dual-energy x-ray absorptiometry, impedance, double-labeled water) might produce erroneous results.

Few studies have explored the segmental distribution of fat and lean stores in people with IBD using more sophisticated techniques. This is of particular importance, as abdominal fat type and distribution have been associated with aggressive 
disease [3] and infectious complications following bowel resection in Crohn's disease [4]. In a recent retrospective study, abdominal myopenia was associated with a primary nonresponse to biologics [5].

Computed tomography (CT) and magnetic resonance imaging (MRI) are routinely undertaken in clinical practice in IBD patients. A CT scan obtained at the third lumbar vertebra allows body compartments such as abdominal skeletal muscle mass and subcutaneous and visceral adipose tissue to be measured directly $[6,7]$.

The primary aim of this study was to assess the abdominal body composition characteristics in a group of IBD patients who had failed medical treatment and underwent a CT scan prior to gastrointestinal surgery. A secondary aim was to explore associations between preoperative abdominal body composition and postoperative outcomes, within a year of IBD surgery.

Table 1 Participants' characteristics

\begin{tabular}{|c|c|c|c|}
\hline Characteristic & IBD $(n=22)$ & Healthy controls $(n=22)$ & P-value \\
\hline \multicolumn{4}{|l|}{${ }^{\star}$ Disease location, $\mathrm{n}$} \\
\hline$L 1$ & 6 & & \\
\hline L2 & 8 & & \\
\hline$L 3$ & 4 & & \\
\hline E1 & 1 & & \\
\hline E2 & 1 & & \\
\hline E3 & 2 & & \\
\hline \multicolumn{4}{|l|}{${ }^{\star}$ Disease behavior, $\mathrm{n}$} \\
\hline$B 1$ & 6 & & \\
\hline$B 2+B 2 p$ & 10 & & \\
\hline$B 3+B 3 p$ & 6 & & \\
\hline \multicolumn{4}{|l|}{ Treatment, $\mathrm{n}$} \\
\hline Aminosalicylates & 13 & & \\
\hline Steroids & 16 & & \\
\hline Thiopurines & 7 & & \\
\hline Biologics & 7 & & \\
\hline \multicolumn{4}{|l|}{ Type of surgery, n } \\
\hline CD; colectomy & 6 & & \\
\hline$C D$; right hemicolectomy & 9 & & \\
\hline CD; left hemicolectomy & 2 & & \\
\hline$C D$; limited small bowel & 1 & & \\
\hline UC; subtotal colectomy & 4 & & \\
\hline Sex, males:females, $\mathrm{n}$ & $12: 10$ & $12: 10$ & $>0.999$ \\
\hline${ }^{\dagger}$ Age, years & $47.5(33: 63)$ & $39.5(30.8: 45.5)$ & 0.045 \\
\hline${ }^{\dagger} \mathrm{BMI}, \mathrm{kg} / \mathrm{m}^{2}$ & $23.6(20.2: 26.8)$ & $27.3(22.8: 34.0)$ & 0.037 \\
\hline${ }^{\dagger}$ Height, $\mathrm{m}^{2}$ & $166.0(156: 175)$ & $171(165: 180)$ & 0.078 \\
\hline${ }^{\dagger}$ Albumin, g/L & $25(23.0: 30.5)$ & & \\
\hline${ }^{\dagger}$ Hemoglobin, g/L & $104.5(98.5: 121.2)$ & & \\
\hline${ }^{\dagger} \mathrm{C}$-reactive protein, $\mathrm{mg} / \mathrm{L}$ & $66(20: 142)$ & & \\
\hline Postoperative complications & 8 & & \\
\hline Wound infection & 5 & & \\
\hline Anastomotic leak & 2 & & \\
\hline Pelvic abscess & 1 & & \\
\hline Number relapsed at 12 months & 4 & & \\
\hline
\end{tabular}

${ }^{*}$ Disease phenotype based on the Montreal classification. ${ }^{\dagger}$ Median (interquartile range)

$I B D$, inflammatory bowel disease; $C D$, Crohn's disease; UC, ulcerative colitis; BMI, body mass index 


\section{Patients and methods}

\section{Participants}

The electronic pathology database from the National Health Service region of Greater Glasgow \& Clyde was searched for all IBD patients who had undergone gastrointestinal surgery for medically refractory IBD between the periods 2012 and 2013. The medical records were then searched to identify patients who underwent a preoperative CT scan of the abdomen and 22 (18 with Crohn's disease and four with ulcerative colitis) were found to have anthropometry measurements available (Table 1). Disease characteristics, disease phenotype, prescribed medication and information on postoperative complications and incidence of clinical relapse within 12 months of operation were collected from the medical notes of the IBD participants (Table 1). Data on the postoperative complications were collected via review of the original surgical notes and notes of any surgical readmissions over the ensuing 12 months. The IBD patients had raised median concentration of C-reactive protein (CRP) and low median serum albumin, indicating an active systemic inflammatory response (Table 1). Two (9\%) IBD patients had a body mass index (BMI) below $18.5 \mathrm{~kg} / \mathrm{m}^{2}$ (underweight) and two (9\%) were obese $\left(\mathrm{BMI}>30 \mathrm{~kg} / \mathrm{m}^{2}\right)$.

A cohort of 22 patients who had CT studies because of acute abdominal pain and in whom no chronic or inflammatory pathology was found was used as a control group. This group was selected from the same pathology database as the IBD group. No one in the control group was underweight but $8(36 \%)$ were obese (Table 1$)$.

As this was a retrospective analysis of existing clinical data, no ethical committee review was required according to National Research Ethics Service guidance [8].

\section{Assessment of body composition}

Abdominal body composition was performed using CT images at the level of the third lumbar vertebra. The images were analysed by two raters independently, as described previously [7]. In brief, the two raters defined the margins of the cross-sectional area $\left(\mathrm{cm}^{2}\right)$ of each abdominal body composition compartment using the freeware program NIH ImageJ (version 1.48). Total fat (excluding intramuscular fat), subcutaneous fat, visceral fat, visceral-to-subcutaneous fat ratio and skeletal muscle cross-sectional area [3] were identified using the Hounsfield unit (HU) thresholds (adipose tissue: -190 to -30 ; muscle: -29 to +150 ) [7]. An example is presented in Fig. 1.

\section{Statistical analysis}

Differences in body composition compartments between the two groups were assessed with forward stepwise multivariate regression analysis. Univariate regression analysis was performed separately for each of the body composition
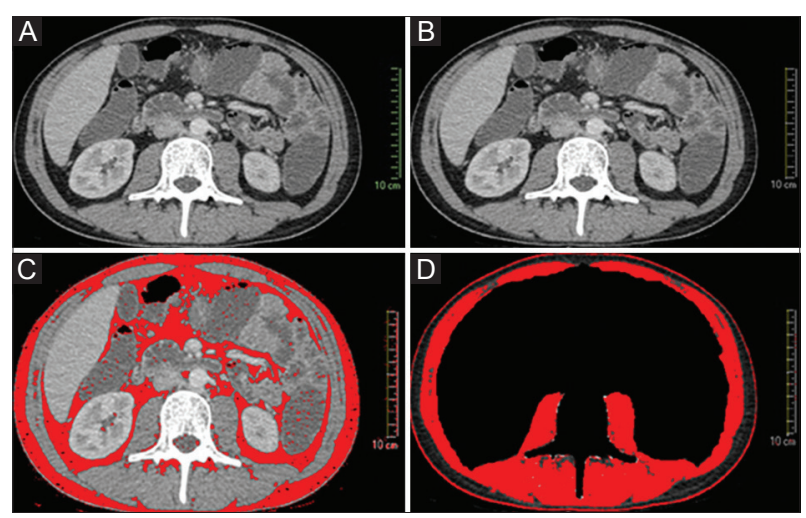

Figure 1 Abdominal body composition analysis using NIH ImageJ. (A) The original CT image; (B) the scale is set at a known distance (10 $\mathrm{cm}$ ) from the original image; (C) definition of the total body fat area, applying the thresholds (-190 to -30 HU); (D) definition of skeletal muscle area, after cropping the abdominal contents and L3 vertebra and applying the thresholds $(-29$ to $+150 \mathrm{HU})$

compartments using predictors selected a priori (height, BMI, age, and sex). The participant's condition (i.e., control or IBD) was a fixed term. Predictors with a P-value $<0.1$ were entered one by one in the multivariate model, starting with the most significant in univariate analysis. A final model was produced that included only significant predictors and the participant's condition (i.e., control or IBD); thus, the independent effect of IBD on body composition could be explored while controlling for the effect of other confounders such as age, height, sex, and BMI. Correlations among composition characteristics were explored using Spearman rho correlation. Associations linking body composition with short-term postoperative complications and a clinical relapse event within 12 months of IBD surgery were explored with logistic regression analysis.

\section{Results}

\section{Predictors of abdominal body composition characteristics and the effect of IBD}

In multivariate analysis, BMI was the strongest positive predictor of visceral and subcutaneous fat, whereas height was the strongest positive predictor of skeletal mass (Table 2). Each unit of BMI increase was associated with a $9.4 \mathrm{~cm}^{2}$ increase in visceral and a $10.7 \mathrm{~cm}^{2}$ increase in subcutaneous fat. No such effect was found for skeletal muscle. Age was positively associated with visceral and subcutaneous fat (Table 2) and sex with skeletal muscle (Table 2). Females had on average $45 \mathrm{~cm}^{2}$ less muscle than males (Table 2).

An independent effect of the participant's condition (i.e. IBD or control) was observed for subcutaneous fat and skeletal muscle but not for visceral fat (Table 2). After accounting for the effect of other confounders, an excess of $38 \mathrm{~cm}^{2}$ for subcutaneous fat and a deficit of $15 \mathrm{~cm}^{2}$ for skeletal muscle mass was observed in IBD people compared with controls (Table 2). There were no significant correlations between the abdominal 
body composition characteristics of the IBD patients with measurements of plasma CRP or serum albumin (all P>0.05).

\section{Relationships among abdominal body composition characteristics}

Relationships between abdominal body composition characteristics are displayed in Fig. 2. Visceral fat was positively correlated with subcutaneous fat for both groups (IBD: rho $=0.62$, $\mathrm{P}=0.002$; controls: rho $=0.61, \mathrm{P}=0.002)$. Abdominal skeletal muscle was positively associated with visceral fat for the control group only (IBD: rho=-0.13, $\mathrm{P}=0.553$; controls: rho $=0.51, \mathrm{P}=0.015)$. While a positive correlation was observed between subcutaneous fat and skeletal muscle in the controls (rho=0.47, $\mathrm{P}=0.026$ ), this relationship was inverted in IBD patients (rho=-0.43, $\mathrm{P}=0.045$ ) (Fig. 2).

\section{Preoperative abdominal body composition characteristics and risk of postoperative complications and subsequent clinical relapse}

Eight participants (38\%) presented postoperative complications, of which the majority were wound infections $(n=5)$, followed by anastomosis leak $(\mathrm{n}=2)$ and pelvic abscess (Table 1). Four patients relapsed within 12 months of operation. None of the abdominal body composition characteristics was predictive of an increased risk of postoperative complications or a subsequent clinical relapse within 12 months of surgery (Table 3).

Table 2 Multivariate regression analysis of predictors of CT-based abdominal body composition characteristics

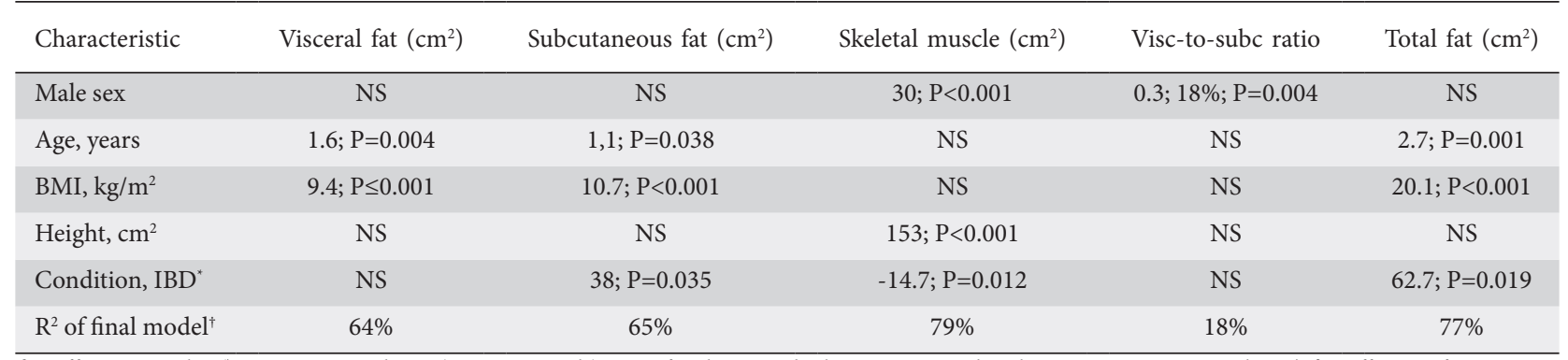

$\beta$ coefficient; P-value. ${ }^{*}$ Participant condition (IBD or controls) was a fixed term in both univariate and multivariate regression analysis. ${ }^{\dagger} R^{2}$ coefficient of determination

Visc-to-subc fat ratio: ratio between visceral and subcutaneous fat area; NS, non-significant; BMI, body mass index; IBD, inflammatory bowel disease
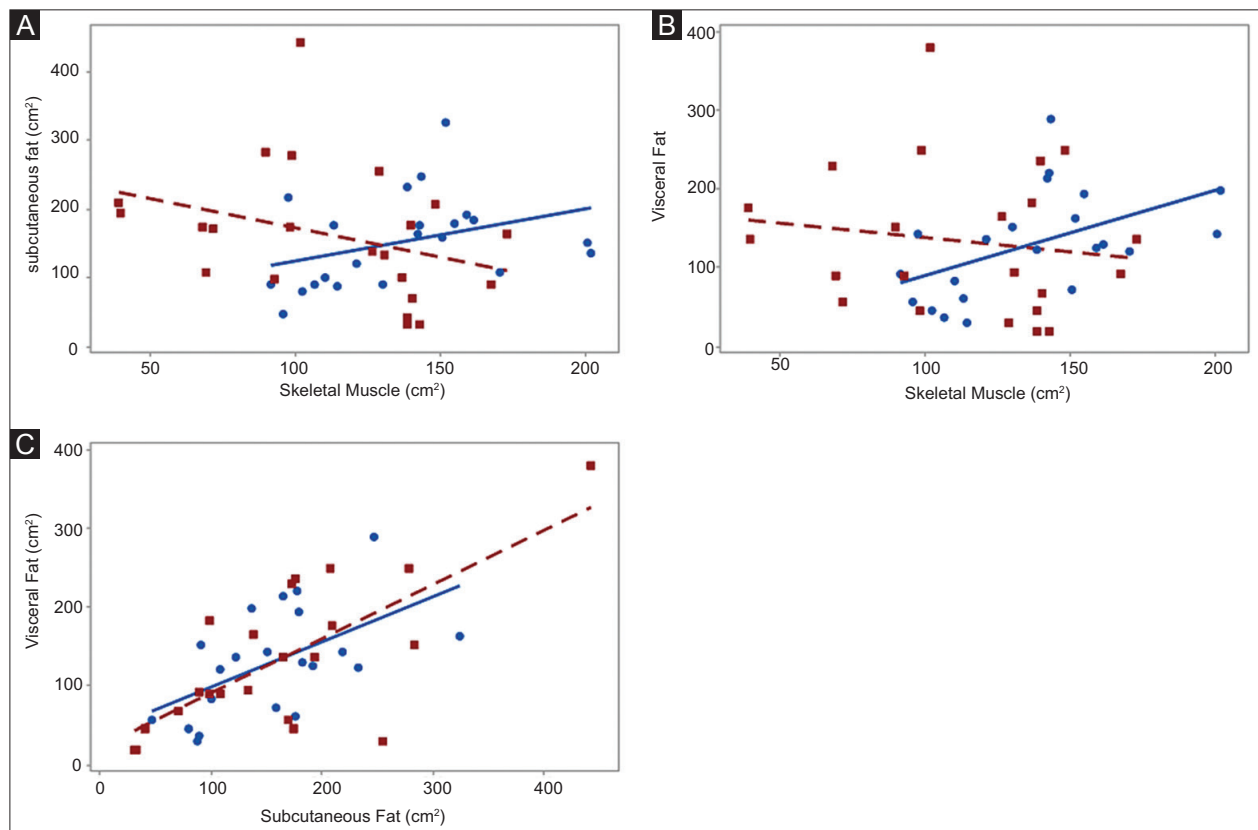

Figure 2 Correlations among abdominal body characteristics in people with inflammatory bowel disease (IBD: brown squares) and controls (blue circles). (A) Subcutaneous fat vs. skeletal muscle: IBD: (rho: $-0.43, \mathrm{P}=0.045)$ vs. controls: (rho: 0.47, $\mathrm{P}=0.026$ ). (B) Visceral fat vs. skeletal muscle: (rho: $-0.13, \mathrm{P}=0.553$ ) vs. controls (rho: 0.51, $\mathrm{P}=0.015$ ); (C) Subcutaneous fat vs. visceral fat; IBD: (rho: $0.61, \mathrm{P}=0.002$ ) vs. controls: (rho: 0.62 , $\mathrm{P}=0.002$ ) 
Table 3 Preoperative abdominal body composition characteristics and risk of postoperative complications and subsequent clinical relapse at 12 months' follow-up

\begin{tabular}{|c|c|c|c|c|}
\hline Characteristic & $\begin{array}{c}\text { Postoperative complications } \\
\text { Odds ratio, } 95 \% C I\end{array}$ & P-value & $\begin{array}{c}\text { Relapse within } 12 \text { months } \\
\text { Odds ratio, } 95 \% C I\end{array}$ & P-value \\
\hline Visceral fat & $1.00(0.99: 1.01)$ & 0.433 & $1.00(0.99: 1.02)$ & 0.386 \\
\hline Subcutaneous fat & $1.00(0.99: 1.01)$ & 0.557 & $1.00(0.99: 1.02)$ & 0.401 \\
\hline Skeletal muscle & $1.00(0.98: 1.02)$ & 0.949 & $0.99(0.96: 1.03)$ & 0.785 \\
\hline Visc-to-subc ratio & $0.69(0.06: 8.6)$ & 0.773 & $1.12(0.06: 21.7)$ & 0.942 \\
\hline Total fat & $1.00(1.00: 1.01)$ & 0.472 & $1.00(1.00: 1.01)$ & 0.372 \\
\hline
\end{tabular}

Visc-to-subc fat ratio: ratio between visceral and subcutaneous fat area

\section{Discussion}

Patients with medically refractory IBD were characterised by deficits in abdominal skeletal muscle mass and an accrual of subcutaneous fat. No alterations were observed with regard to visceral fat. Together, these abdominal body composition characteristics are suggestive of features of nutritional cachexia similar to those seen in other inflammatory conditions, such as cancer [9], and can be attributed to undernutrition, the effect of proinflammatory cytokines and steroid treatment [10].

The relationship seen in controls between skeletal muscle and the two adipose tissue compartments was absent or reversed in IBD. The exact mechanism of this association is unknown, but it is likely to be multifactorial and to involve diminished physical activity in patients with active IBD, the effect of inflammatory cytokines on muscle mass [11] and protein metabolism [12], the excessive use of steroids or a primary role of visceral fat in the initiation of colonic inflammation $[13,14]$. A previous study showed that normalization of BMI at 2-year follow up was not associated with an increment in fat-free mass in patients with Crohn's disease [15]. Collectively, this evidence suggests that a change in BMI might not be a good proxy for body alterations in IBD.

The clinical significance of these results should also be evaluated in the context of their ability to predict clinical outcomes. Previous research suggested that the visceral-tosubcutaneous fat ratio may be associated with postoperative complications [4] and disease severity [3], but that finding was not replicated in this small retrospective study of patients with medically refractory disease, prior to gastrointestinal surgery. Patients with IBD are at higher risk of adverse cardiovascular events [16] than the general population and, based on the current findings, compared with healthy individuals they should be expected to have a higher degree of abdominal adiposity for a given BMI. Thus, clinical attention should be given to the avoidance of overnutrition, particularly now that the epidemic of obesity has made it common in IBD [17]. It might be that the focus on the dietetic support of IBD patients should be extended from the management of undernutrition to the prevention and correction of obesity, as well as interventions with physical activity and exercise to improve muscle mass.

The strength of this study is the inclusion of an essentially healthy control group for first time $[3,4]$, as well as the direct and independent assessment of each of the abdominal

\section{Summary Box}

\section{What is already known:}

- Patients with inflammatory bowel disease (IBD) often have altered body composition

- The type and distribution of abdominal fat has been associated with complicated disease and poor postoperative outcomes in patients with IBD

\section{What the new findings are:}

- In patients with treatment refractory IBD, abdominal body composition is characterized by excessive fat deposition and skeletal muscle deficits

- Physiological relationships between skeletal muscle mass and subcutaneous and visceral abdominal fat do not apply in IBD or are inverted in such patients

- A larger body size for the same anthropometry should be anticipated in patients with IBD compared with healthy controls

- Preoperative abdominal body composition is not predictive of postoperative outcomes

compartments. This is a major advantage compared to previous studies in IBD, where measurement error in one body compartment might have propagated to the estimation of the others [1]. The main limitations are the small sample size, the heterogeneous patient population in terms of disease characteristics, and the retrospective design of the study. Use of concomitant treatment following surgery may also have influenced the risk of postoperative complications and subsequent clinical relapse. Moreover, our control group was younger and had a higher BMI than our IBD patients, which may have confounded the results of this study. However, instead of stratifying our analysis by these confounders, which would have further compromised statistical power and increased bias due to multiple testing, we chose to apply multivariate regression modelling to control for their effect. A lack of a biological association between adipose tissue and sex was not observed, but not unexpectedly, as our analysis was performed 
in a cross-section of the abdomen and expressed in units of area, rather than as a percentage of the total area.

In conclusion, abdominal body composition characteristics in this study highlight the incidence of sarcopenia in medically refractory IBD patients. The significance of these findings in terms of clinical disease course and long-term outcomes of IBD should be explored in future prospective studies, particularly now that radiation-free MRI assessment is becoming more accessible and affordable. This study has clear implications for the nutritional assessment and management of people with IBD.

\section{References}

1. Bryant RV, Trott MJ, Bartholomeusz FD, Andrews JM. Systematic review: body composition in adults with inflammatory bowel disease. Aliment Pharmacol Ther 2013;38:213-225.

2. Azcue M, Rashid M, Griffiths A, Pencharz PB. Energy expenditure and body composition in children with 'Crohn's disease: effect of enteral nutrition and treatment with prednisolone. Gut 1997;41:203-208.

3. Erhayiem B, Dhingsa R, Hawkey CJ, Subramanian V. Ratio of visceral to subcutaneous fat area is a biomarker of complicated 'Crohn's disease. Clin Gastroenterol Hepatol 2011;9:684-687.

4. Stidham RW, Waljee AK, Day NM, et al. Body fat composition assessment using analytic morphomics predicts infectious complications after bowel resection in 'Crohn's disease. Inflamm Bowel Dis 2015;21:1306-1313.

5. Ding NS, Malietzis G, Lung PFC, et al. The body composition profile is associated with response to anti-TNF therapy in 'Crohn's disease and may offer an alternative dosing paradigm. Aliment Pharmacol Ther 2017;46:883-891.

6. Mourtzakis M, Prado CM, Lieffers JR, Reiman T, McCargar LJ, Baracos VE. A practical and precise approach to quantification of body composition in cancer patients using computed tomography images acquired during routine care. Appl Physiol Nutr Metab 2008;33:997-1006.

7. Richards CH, Roxburgh CS, MacMillan MT, et al. The relationships between body composition and the systemic inflammatory response in patients with primary operable colorectal cancer. PLoS One 2012; 7:e41883.

8. Available from: https://researchsupport.admin.ox.ac.uk/sites/ default/files/researchsupport/documents/media/definingresearch.pdf. [Last accessed on 2018 May 18].

9. Peng PD, van Vledder MG, Tsai S, et al. Sarcopenia negatively impacts short-term outcomes in patients undergoing hepatic resection for colorectal liver metastasis. HPB (Oxford) 2011;13:439-446.

10. Gerasimidis K, McGrogan P, Edwards CA. The aetiology and impact of malnutrition in paediatric inflammatory bowel disease. J Hum Nutr Diet 2011;24:313-326.

11. Figueiredo VC, Markworth JF, Durainayagam BR, et al. Impaired ribosome biogenesis and skeletal muscle growth in a murine model of inflammatory bowel disease. Inflamm Bowel Dis 2016;22:268-278.

12. Zoico E, Roubenoff R. The role of cytokines in regulating protein metabolism and muscle function. Nutr Rev 2002;60:39-51.

13. Büning C, von Kraft C, Hermsdorf $M$, et al. Visceral adipose tissue in patients with 'Crohn's disease correlates with disease activity, inflammatory markers, and outcome. Inflamm Bowel Dis 2015;21:2590-2597.

14. Paeschke A, Erben U, Kredel LI, Kühl AA, Siegmund B. Role of visceral fat in colonic inflammation: 'from Crohn's disease to diverticulitis. Curr Opin Gastroenterol 2017;33:53-58.

15. Sylvester FA, Leopold S, Lincoln M, Hyams JS, Griffiths AM, Lerer T. A two-year longitudinal study of persistent lean tissue deficits in children with 'Crohn's disease. Clin Gastroenterol Hepatol 2009;7:452-455.

16. Singh S, Kullo IJ, Pardi DS, Loftus EV Jr. Epidemiology, risk factors and management of cardiovascular diseases in IBD. Nat Rev Gastroenterol Hepatol 2015;12:26-35.

17. Flores A, Burstein E, Cipher DJ, Feagins LA. Obesity in inflammatory bowel disease: a marker of less severe disease. Dig Dis Sci 2015;60:2436-2445. 